\title{
Substudy Details
}

National Cancer Institute

\section{Source}

National Cancer Institute. Substudy Details. NCI Thesaurus. Code C126075.

A textual description of the substudy. 